\title{
The attainment of physician's professional identity through meaningful practice: A qualitative study
}

\author{
Maryam Shahabi ${ }^{1}$, Nooredin Mohammadi², Jalil Koohpayehzadeh ${ }^{3}$, Seyed Kamran Soltani Arabshahi*1(1) \\ Received: 18 Aug 2018 \\ Published: 5 Mar 2020
}

\section{Abstract}

Background: Medical professional identity is how an individual perceives him/herself as a doctor. Formation of professional identity includes development, advancement, and socialization through social learning of specific knowledge, skills obtained while performing professional roles, practicing, and new attitudes and values. A qualitative study was performed to examine live experience of undergraduate medical science students with regards to obtaining professional identity.

Methods: This qualitative study was performed using a conventional content analysis method. Participants were students who were studying medicine at Iran University of Medical Sciences. Sampling was done based on a purposeful sampling method. A total of 23 students took part in semi-structured interviews until data saturation was reached. The interviews were transcribed verbatim. Also, to develop themes, data were analyzed using conventional content analysis. Moreover, data management was done using MAXQDA software.

Results: Based on data analysis, 2 main themes were as follow: meaningful medical practice" and "professional medical practice". The first theme had 3 categories: (i) self-insight; (ii) manner; and (iii) values and beliefs. The second theme had 2 categories: (i) professionalism; and (ii) holistic view of medicine.

Conclusion: Certain individual characteristics and personality type were factors that affected participants' choice of their field of study. The participants' understanding of their profession was formed, not only by studying in the university through learning relevant knowledge, skills, and practice, but also by perceived attitude, views, and values in their profession.

Keywords: Qualitative study, Professional identity, Physician, Medical education

Conflicts of Interest: None declared

Funding: Iran University of Medical Sciences

\section{*This work has been published under CC BY-NC-SA 1.0 license.}

Copyright $\odot$ Iran University of Medical Sciences

Cite this article as: Shahabi M, Mohammadi N, Koohpayehzadeh J, Soltani Arabshahi SK. The attainment of physician's professional identity through meaningful practice: A qualitative study. Med J Islam Repub Iran. 2020 (5 Mar);34:16. https://doi.org/10.47176/mjiri.34.16

\section{Introduction}

Professional identity is a serious issue in medical education, and the advancement of professional ethics is rooted in professional identity. The Royal College of Physicians defines professionalism as follows: "A set of
Corresponding author: Dr Seyed Kamran Soltani Arabshahi, soltarab@iums.ac.ir

1. Center for Educational Research in Medical Sciences (CERMS), Department of Medical Education, School of Medicine, Iran University of Medical Sciences, Tehran, Iran.

2. Department of Critical Care Nursing, Nursing and Midwifery Faculty, Nursing Care Research Center, Iran University of Medical Sciences, Tehran, Iran.

3. Community Medicine Department, CERMS (Center for Educational Research in Medical Sciences), Faculty of Medicine, Iran University of Medical Sciences, Tehran, Iran. $\uparrow$ What is "already known" in this topic:

- Since professional identity is a culture-based concept, and its definition is influenced by a variety of factors, it should be considered separately in each country and culture.

- Some attributes make up medical professional identity

- Ethics is one of the features of professional identity.

$\rightarrow$ What this article adds:

- This article introduces new views and approaches with regards to physician's identity

- Medical professional identity based on the experiences of intern students and general physicians were found to be meaningful medical practice

Manner, self-insight, beliefs and value, professionalism, and holistic approach were some characteristics of medical professional identity in ethical domain. 
values, behaviours, and relationships that enables society to trust physicians" (1). The American Board of Internal Medicine, American College of Physicians, and European Federation of Internal Medicine came together and tried to provide a functional definition for professionalism that is able to reflect the realities of contemporary times. In this regard, they identified 3 fundamental principles as the basis for professionalism: (1) a patient-centred approach rather than a disease-centred approach, (2) patient autonomy and social justice; (3) and self-perceived professional or professional identity (2).

The issue of professional identity started to become important when the disease-centred approach faced criticism. With the emergence of technological and technical development in medicine within the last century, it became customary for medical schools to teach the treatment of diseases based on the biomedical paradigm. This paradigm is knowledge-based and disease-centred, which does not take into account the many individual and social factors involved. This approach has now faced various challenges, because medicine is not exclusively about the disease itself. It is also about the preservation and improvement of human health. Human beings consist of body and soul. Therefore, any therapeutic intervention on the body of an individual will also affect their soul and mental health. A physician who does not pay attention to this fact is no different than a computer program that interprets and analyses the patient's test results (3). Medical students should accept a set of values, characteristics, and behaviours that enables the society to trust and have confidence in the medical profession, as part of the concept of medical professionalism. Moreover, they should put this into practice and be able to make appropriate decisions, while respecting the legal requirements and code of ethics and the culture and beliefs of the patients. Therefore, they must have a clear understanding of their professional identity, and use this as a guide for their professional conducts and decision-making. This will not be possible without a professional identity $(4,5)$.

The expansion of social life has caused the issue of professionalism and professional identity to become broader and more complex. Moreover, it has become more difficult to detect ethical instances from nonethical ones. This dilemma highlights the importance of the physician's commitment to professional principles and values and professional identity. There is no doubt that this commitment can be found in the physician's understanding of professional identity and the formation of a professional characteristic during their medical education and training (6). Creating and influencing professional identity, personality, and its associated professional behaviours and professionalism in general practitioners is one of the issues that has caught the attention of medical schools. Moreover, the value of such work has been mentioned in numerous studies and literature (7). Professionalism is one of the competences that physicians must have because of the critical nature of their occupation, given the fact that they are dealing with human beings. Professionalism means having manners and professional behaviours. This is beyond a series of principles, rules, and ethical codes that one must follow. Therefore, for physicians to feel inclined and committed to conduct themselves in a professional behaviour, they must first have an understanding of what their professional identity entails and shape their personality traits and characteristics in a way that their identity integrates benevolence and professionalism when dealing with patients.

Thus, the clarification of the meaning of professional identity in medicine is a way of promoting professionalism and development. This provides a context for professional development, social accountability, and offering better services, and thereby increasing patient satisfaction (8). One of the ultimate goals of medical education is to enable students to attain professional identity; in other words, the development of a distinct entity from the concept of "a good physician" (9). This is more than being academically qualified and involves changing students' perspective and attitude with regards to morals, values, and professional behaviour (10). However, there are still those who support the view of Aristotle that such qualities cannot be taught, because the individual is born with them (11). However, there is contradictory evidence with regards to this matter (12).

Wald defines medical education as a peculiar event in which a lay person transforms into a professional individual in care by gaining the necessary knowledge, skills, attitudes, values, and characteristics. In other words, it is defined as education in its broadest sense (13). Hafferty and Frank also consider medical education as a transformation process in which a person becomes a doctor and the values of this new culture, along with strategies and techniques, are taught to new students. Moreover, students are provided with opportunities that allow them to internalize these values and the concept of professional identity is formed within these individuals (14). How is professional identity formed? The medical culture values and the perception that "who is the ideal physician?" quickly influences and develops the students' clinical experiences in classrooms and laboratories $(15,16)$. Despite the importance of the issue, role, and effect of medical professional identity in providing qualify services, the concept of medical professional identity in Iran has not been properly recognised. Addressing the issue of professional identity and professional development in the field of medical education in Iran is almost new. In addition, it has not been satisfactorily addressed in published literature in Iran. Since the concept of medical professional identity roots from individuals' point views and experiences, it is necessary to explore the meaning of the concept in this specific society. Therefore, explaining the features and attributes of professional identity is one of the first essential steps that can advance medical education and improve the Iranian health care system. The purpose of this study was to explore the attainment of professional identity from physicians' perspectives.

\section{Methods}

This was a qualitative content analysis which intended to explain the concept of medical professional identity. The Landman and Graneheim method for content analysis 
was used. The use of qualitative studies and methods is recommended when the purpose of a research, in addition to describing behaviour, is to uncover and explain. Such studies are useful when the views and attitudes of the target community in relation to the subject matter is not clear, or the information available is insufficient. Moreover, in certain cases, there are no suitable terms for communicating with the audience (17). Qualitative research provides the possibility of examining the underlying behaviours, attitudes, and perceptions that determine the health status of individuals.

The participants of this study included 23 interns (internship student) and general practitioners from medical schools. The data collection method in this study was semi-structured face to face interviews. After obtaining permission from the participants to use a tape recorder, the researchers conducted the interview sessions in a quiet, appropriate room. The method used for collecting samples was purpose-based and with maximum variation. The interviews continued until data saturation and extraction of themes and classes were reached. The researchers used example questions to help them when conducting interviews. Some of the examples used included "How do you describe the identity of a doctor?", "A doctor is someone who...?".

"Based on your lived experience, when a doctor is qualified for this profession?". Then, further questions were asked to explore the factors affecting the definition of the concept of medical professional identity, while taking notes at the same time. The participants enrolled in the study voluntarily and were able to leave the study at any time during the course of the study. Each of the interviews lasted for 45-60 minutes. Before starting the interviews, the participants were informed about the purpose of the study and were assured that their information will remain strictly confidential.

For analysis, after repeatedly listening to the recordings, the whole text of the interviews was transcribed word by word and encoded. For data analysis, audio files from the interviews were converted into text first. Simultaneously with the start of the first interview, the texts from the audio files of the interviews were coded and analysed using content constant comparison. The interviews were listened by the principal investigator for several times until an overall idea was obtained and ambiguities were resolved by checking the transcript data with participants soon after the interview or simultaneously at the time of interview. Then, the meaning units and parts of data, which were directly related to the research question, were identified. Subsequently, the data analysis was performed based on Graneheim et al (17).
To verify the accuracy of the research, attempts were made to increase the validity of the research findings using the following strategies: having multiple in-depth interview sessions in different situations at the participants' convenience, combining multiple data collection methods such as observing and taking notes in the field, variation in the selection of participants, member check by the participants to verify the data $(18,19)$, peer debriefing, and peer check of the data by the supervisors and colleagues to ensure that the categories were consistent with the statements of the participants (20). To obtain the approval of the study, the interviews, codes, and categories that were extracted were all analysed by the research team and the researcher. At the same time, the texts of some of the interviews, codes, and categories, and subcategories extracted were given to a number of experts and colleagues to evaluate the agreement made regarding the meanings between several researchers (21).

This study was approved by the Ethics Committee of Iran University of Medical Sciences. Also, oral and written informed consent was obtained from participants before participation in the study and recording of the interviews. Participants were free to enter and withdraw from the study at any time. They were assured of the confidentiality of the information and were allowed by the researcher to have phone calls and send e-mails regarding any questions or information.

\section{Results}

The 2 main components of meaningful medical practice and professional medical practice were derived from data analysis (Table 1).

The following sections elaborate on the categories extracted from the interviews:

\section{Meaningful medical practice}

Meaningful medical practice was the first category extracted from the data analysis process. Meaningful medical practice reflects the fact that the individuals who are suitable for this profession are those who believe that meaningful practice is esoteric. In other words, one has meaning and philosophy in their work. That is, human beings are constantly seeking meaning and purpose for what they are doing. This category consists of 3 subcategories, including manner, self-insight, and values and beliefs.

1.1. Manner: This subcategory represents one of the main attributes, which is important for the participants. The codes for this subcategory describe the features that are essential for obtaining medical identity. The concepts that were frequently used in the interviews were a sense of value through doing professional responsibility, compas-

Table 1. Main theme, categories and sub-categories

\begin{tabular}{lcc}
\hline Main theme & Categories & Subcategories \\
\hline & Meaningful medical practice & Manner \\
Medical identity Attributers & & Value and believe \\
& Professional medical practice & Professionalism \\
& & Holistic approach \\
\hline
\end{tabular}


sion, being of assistance and help, desire to provide service, patience, high endurance, and prioritise the right of the patient.

Hence, one of the characteristics of the medical profession's identity, which can be challenging and varied, is their individuality. One of the participants stated, "Being a physician is a lifestyle and not just a job. One must prioritise this aspect and it should not be a goal to reach, but a way of living." (Participant 8). Another participant stated, "Generally, a good physician is first and foremost a good person. That is, the person they are dealing with should be important to them." (Participant 2).

1.2. Self-insight: The codes of this subcategory represent the importance of having this feature in this profession to be able to provide efficient and effective specialised services. Every individual has to know how to serve as one member of health care team. An insight needs to be targeted and enhanced to improve the skills of the individuals and improve nurturing. A vision needs to be worth the effort one has to put in, and only the insight of participants can determine the path. One of the biggest concerns that may undermine the quality of service that a physician provides is having to answer his own questions regarding what he is doing. One of the participants believed that, "This was a way of thinking and looking at things that one must have in the field of medicine to be successful, which means that you have to think, feel, and act like a physician." (Participant 4).

1.3. Beliefs and values: Another feature within the context of meaningful medicine is values and beliefs. Values are, in fact, the criteria which measure the correctness of one's path. Without values, perhaps one may develop insights that are unethical. However, values cannot determine the path, only insight can do this. Inner belief, inner love, and inner faith towards the path and purpose were mentioned by interviewees. One of the participants in this field stated, "In my opinion, if a doctor is not certain about the path they have chosen, they will never be able to overcome and endure the difficulties they face." (Participant 21). Another participant said, "If a person does not have purpose in life, they won't know what's important to them and won't know what their personal values are." (Participant 18).

\section{Professional medical practice}

Professional medical practice is the second category extracted from the data analysis process, which represents a general characteristic for the development of medical identity and work in this field. Based on the findings of this study, medicine is a profession that only appears in full form when all its aspects are acknowledged. Professionalism and a holistic approach are among the main subcategories that indicate professionalism in medicine. Further details regarding these subcategories are provided using direct quotations from the participants.

2.1. Professionalism: Medical professionalism is " $A$ set of attitudes and behaviours in medical students and physicians, which causes the individual to always put the patient's interest above their own personal interests." (22). This subcategory includes codes related to the foun- dations and ethical and organisational rules of the medical profession, where each physician who is interested in providing professional services, by acquiring them, can work in this field. One of the participants stated, "We have special programs to monitor the professors. For example, evaluation of the professors and self-assessment that are not really valid, but by achieving professional identity, if it is properly institutionalised within these individuals, it can act as a great form of self-care and self-control. The individual will be determined and feel responsible to perform their professional duties properly without the need for external pressure. In this case, all of these external monitoring and supervisions will become extra and unnecessary." (Participant 16). Another participant believed that, "Teaching professionalism should be a longitudinal theme in the curriculum. Through repetition, it becomes internalised within the individual and becomes a part of their identity and personality. In this case, we have been successful in educating our medical students." (Participant 19).

2.2. Holistic approach: A holistic approach is one of the required characteristics and behavioural features for physicians. Another issue that contributes to professional medical practice is a holistic view of medicine. From the perspective of many psychologists, humans are whole beings. Moreover, human beings are multidimensional beings and ignoring any of the dimensions essentially ignores vital parts of their humanity (23) .Accordingly, human action is influenced by all aspects of their existence. If we consider the disease as an action, it is obvious that this action is influenced by its biological, psychological, social, and spiritual dimensions. Therefore, in addition to the knowledge and skills that they are required to have about human physiology health professionals, they also need accurate information on the spiritual issues of their patients $(24,25)$. Moreover, they need to treat patients with a patient-centred approach rather than a diseasecentred method. The participants believed that the holistic approach emerged to compensate for the diminutive approach of modern medicine, which seeks to explain human disease with biological concepts. This school of thought is also trying to integrate social, cultural, and personal experiences into medicine. One of the participants believed that, "There is a medical approach that is called holistic medicine. Spirituality was shaped and entered medicine from this approach. This approach suggests that a person should be looked at as a whole being to change the way medicine is currently being practiced." (Participant 10).

\section{Discussion}

This study addresses the emerging issue of the acquisition and development of professional identity in general practitioners in Iran. Because our information in this area is limited and ambiguous, we used the perspective and experience of the participants to investigate this matter. Using this method, one can first and foremost learn about the nature of medical identity and use a strategic tool to discover the behaviours, goals, individual and interpersonal interests, present and future threats, and opportuni- 
ties. This will help improve the quality of education, and in particular, the upbringing and training of future physicians. The findings of this study reflect the changes made and the long path to gaining and developing professional identity in medical students. The results of data analysis showed what characteristics lead to the acquisition of medical professional identity. What scientific knowledge and practical qualification should eligible individuals have? More importantly, what are the personality and behavioural features required to become a physician and acquire medical identity? By developing effective attitudes and training such forces and, consequently, by delivering quality and efficient health services, we can promote accountability in medicine and increase the general health of society. In the extracted material from this study, it was stated that individual personality, individual insight, beliefs, and values in medicine and the physician contribute significantly to the professional life of physicians and meaningful medical practice. Meaningful work and profession mean believing in goals and values, as well as caring about the quality of the work and professional life and understanding the value of professional performance.

The findings of this study showed that one of the important attributes of becoming a physician and transitioning from being an ordinary person to becoming a physician is having a personal disposition, personal insight, vision, and philosophy in the profession, and believing in your profession. These findings reflect the changes medical students experience during their years of study and training they undergo for professional development and professionalism. In addition, they can enter the medical profession by understanding the nature of this profession, accepting the lifestyle that comes with it, and having an understanding of who they are and their profession. One of the characteristics necessary for acquiring identity and meaningful careers is having a personal disposition. In fact, acquiring personal character means that medical students develop the qualities that are essential to the medical profession. For example, a sense of value through personal responsibility, goodwill, providing services, resilience, patience, tolerance, morality, prioritizing the patient were some of the cases referred to by physicians and interns. Moreover, intrinsic motivation to continue the long and difficult path to the medical field and to feel valued by doing professional work and giving value to the cause and purpose of the service were mentioned by most practitioners and undergraduates as meaningful medical practice. Philosophy recognizes one's self, existence, and values, shaping individual decisions and behaviors. Philosophy defines beliefs and values and the way people interact with one another and is the set of beliefs and values that guide and shape one's behavior. Philosophy discussion and the need to be aware of its dimensions in our relationships, behaviors, and ethical decisions emerge from the medical sciences, physicians, and nurses as providers of health care in their relationships with humans $(26,27)$.

This term has been introduced mostly with purpose, value, and belief about the journey of life (28). The values of an individual are associated with all aspects of their life, guiding the daily routine of their life, as well as being a source of support, strength, and movement in their journey. In fact, spirituality is an individual effort to achieve a special understanding of life's purpose and meaning (29). Based on the perspective of many psychologists, humans are whole beings. Furthermore, human behaviour is influenced by all aspects of his existence, and in particular, his attitude acquires meaning and direction. Therefore, health care professionals, especially physicians, need to approach their profession with a certain attitude, which itself directs one's behaviour, manner, and approach taken when dealing with issues, in addition to the knowledge and skills required regarding human physiology (30). Spiritual vision and thinking, developing self-awareness, forming spiritual identity, developing identity, and developing professional practice is a way of addressing clinical issues with a different perspective $(31,32)$. Frankel stated that meaning comes from specific individual achievements, as well as their interactions with the immediate world. Confusion, and loss of meaning in life, reflects the decline in the sense of value of life and suggests that life is a negative experience. In such a case, there is no incentive to pursue important goals, and the individuals become confused about themselves and life (33).

\section{Conclusion}

Certain individual characteristics and personalities affected the tendency of participants to choose this profession. Professional identity was formed, not only by studying in the university through learning relevant knowledge, skills, and practice but also with perceived attitude, views, and value in their profession.

Professional identity is one of the features of an individual that can be defined as personal satisfaction and a practical commitment to the assigned tasks within their profession, provided that the individual is doing their job in the best way possible without having any supervisory system in place. Teaching the features of professional identity to physicians can enhance professionalism, which can ultimately enhance the quality of health care services.

It is suggested that the prescriptive model of medical professional identity in the context of Iran be performed by future research which can be tested by measuring these effects on performance of physicians. Moreover, due to the limited sampling environment to Tehran, the findings should be interpreted with caution.

\section{Conflict of Interests}

The authors declare that they have no competing interests.

\section{References}

1. Tallis RC. Doctors in society: medical professionalism in a changing world. J Clin Med. 2006;6(1):7-12.

2. ABIM Foundation. American Board of Internal Medicine; ACPASIM Foundation. American College of Physicians-American Society of Internal Medicine; European Federation of Internal Medicine. Medical professionalism in the new millennium: a physician charter. Ann Intern Med. 2002;136(3):243.

3. Elcin M, Odabasi O, Ward K, Turan S, Akyüz C, Sayek I. The first medical humanities programme in Turkey. Int $\mathrm{J}$ Med Educ. 2006;40(3):278-82

4. Wachtler C, Lundin S, Troein M. Humanities for medical students? A 
qualitative study of a medical humanities curriculum in a medical school program. BMC Med Educ. 2006;6(1):16.

5. Hadizadeh F, Yazdani S, Ferdosi M, Haghdoost AA, Rashidian A, Hadadgar A, et al. How can we motivate medical sciences students to learn health system management? A report of the first national Olympiad on managerial reasoning and decision-making. Int J Res Med Sci. 2012;17.

6. Yamani N, Changiz T, Adibi P. Professionalism and hidden curriculum in medical education. Isfahan: Isfahan University of Medical Science, Medical Education Research Center [citd 2013 Dec 19] available from: rds sem-ums ac ir/edc/downloads/professionalism and hidden pdf. 2004.

7. Brott PE, Myers JE. Development of professional school counselor identity. Prof Sch Couns. 1999;2(5):339-48.

8. Lake CL. Professionalism: the creation, promotion, and maintenance of professional behavior. Adv Anesth. 2005;23:1-14.

9. Irby D. Educating physicians for the future: Carnegie's calls for reform. Med Teach. 2011;33(7):547-50.

10. Monrouxe LV. Identity, identification and medical education: why should we care?. Med Educ. 2010;44(1):40-9.

11. Finn G, Garner J, Sawdon M. 'You're judged all the time!'Students' views on professionalism: a multicentre study. Med Educ. 2010;44(8):814-25.

12. Hodges BD, Ginsburg S, Cruess R, Cruess S, Delport R, Hafferty F, et al. Assessment of professionalism: recommendations from the Ottawa 2010 Conference. Med Teach. 2011;33(5):354-63.

13. Wald HS, Anthony D, Hutchinson TA, Liben S, Smilovitch M, Donato AA. Professional identity formation in medical education for humanistic, resilient physicians: pedagogic strategies for bridging theory to practice. Acad Med. 2015;90(6):753-60.

14. Hafferty FW. Beyond curriculum reform: confronting medicine's hidden curriculum. Acad Med. 1998;73(4):403-7.

15. Chuang AW, Nuthalapaty FS, Casey PM, Kaczmarczyk JM, Cullimore AJ, Dalrymple JL, et al. To the point: reviews in medical education-taking control of the hidden curriculum. Am J Obstet Gynecol. 2010;203(4):316. e1-. e6.

16. Holden M, Buck E, Clark M, Szauter K, Trumble J, editors. Professional identity formation in medical education: the convergence of multiple domains. HEC forum; 2012: Springer.

17. Graneheim UH, Lundman B. Qualitative content analysis in nursing research: concepts, procedures and measures to achieve trustworthiness. Nurse Educ Today. 2004;24(2):105-12.

18. Holloway I. Qualitative research in health care: McGraw-Hill Education (UK); 2005.

19. Tolley EE, Ulin PR, Mack N, Succop SM, Robinson ET. Qualitative methods in public health: a field guide for applied research: John Wiley \& Sons; 2016.

20. Creswell JW, Creswell JD. Research design: Qualitative, quantitative, and mixed methods approaches: Sage publications; 2017.

21. Malterud K. Qualitative research: standards, challenges, and guidelines. Lancet. 2001;358(9280):483-8.

22. Saberi A, Nemati S, Fakhrieh Asl S, Heydarzadeh A, Fahimi A. Education of Medical Professionalism and the Role of Educators of Guilan University of Medical Sciences, Iran, According to its Residents. Strides Dev Med Educ. 2013;10(2):218-24.

23. Kachoie A, Ahmari Tehran H, Dehghani F, Abbasi M, Parizad A. Physicians' Attitudes towards Spirituality and Pastoral Care. J Mazandaran Uni Med Sci. 2016;26(138):151-60.

24. Adolphs R, Tranel D, Damasio AR. Dissociable neural systems for recognizing emotions. Brain Cogn. 2003;52(1):61-9.

25. Dowling EM, Gestsdottir S, Anderson PM, Von Eye A, Almerigi J, Lerner RM. Structural relations among spirituality, religiosity, and thriving in adolescence. Appl Dev Sci. 2004;8(1):7-16.

26. Miller CA. Nursing for wellness in older adults: Theory and practice: Lippincott Williams \& Wilkins.Acad Med; 2004.

27. Meleis AI. Theoretical nursing: Development and progress: Lippincott Williams \& Wilkins. Acad Med; 2011.

28. Buck HG. Spirituality: concept analysis and model development. Holist Nurs Pract. 2006;20(6):288-92.

29. McKenna H. Nursing theories and models: Routledge; 2006.

30. McSherry W, Jamieson S. An online survey of nurses' perceptions of spirituality and spiritual care. J Clin Nurs. 2011;20(11-12):1757-67.

31. O'Brien ME. A nurse's handbook of spiritual care: standing on holy ground: Jones \& Bartlett Learning(JBL); 2004.

32. Singh DKM, Ajinkya S. Spirituality and religion in modern

medicine. Indian J Psychol Med .2012;34(4):399.

33. Frankl VE. Man's search for meaning: Simon and Schuster; 1985. 